\title{
Modules and Bounded Linear Operators
}

\author{
Muna Jasim and Manal Ali \\ Department of Mathematics, College of Science for Women, Baghdad University.
}

\begin{abstract}
An associated R-module of $T$, which is denoted by $V_{T, T^{*}}$ is given, Where $V$ is an inner product space and $T$ is bounded linear operator on $V$. We study in this paper properties of $T$ which effects $V_{T, T^{*}}$ and conversely.
\end{abstract}

Keywords: The module of an operator, algebraic elements of $T$, torsion elements of $H_{T, T^{*}}$.

\section{Introduction}

SALMA M. FARIS in [1] described a left R-module $V$ where R the polynomials ring in $\mathrm{x}$ and $V$ is vector space as follows:- $\emptyset: R \times$ $V \rightarrow V \quad$ by $\varnothing(P, v)=P . v=P(T) v \quad$ this function makes $V$ a left $R$-module denoted by $V_{T}$. In this paper we start by introducing a left $\mathrm{R}$ - module on the ring of polynomials in $\mathrm{x}, \mathrm{y}$ and $V$ is an inner product space as follows:$\Psi: R \times V \rightarrow V$ by $\Psi(P, v)=P\left(T, T^{*}\right) v$ this function makes $V$ a left $R$-module and denote this module by $V_{T, T^{*}}$.In proposition (3.2) we give form of elements of $V_{T, T^{*}}$. We prove that $V_{S, S^{*}}$ is isomorphic to $V_{T, T^{*}}$ if and only if $S$ is similar to $T$, we study the relation between the *- algebraic elements and the torsion elements of $H_{T, T^{*}}$, and the module associated with the unilateral shift operator we prove that $H_{U, U^{*}}$ is acyclic R-module.

\section{Preliminaries}

In this section the fundamental basic concepts and primitive results are Given.

\section{Definition (2.1) [1]:}

Let $V$ be a vector space over a field $F$. Let $T$ be a linear operator acting on the elements of $V$ on the left .Let $\mathrm{R}=\mathrm{F}[\mathrm{x}]$ be the ring of polynomials in $\mathrm{x}$ with coefficients in $\mathrm{F}$. Define $\emptyset: R \times V \rightarrow V$ by $\emptyset(P, v)=P . v=$ $P(T) v$.

It is clear that $\varnothing$ makes $V$ a left $R$-module denoted $V_{T}$, and call it the associated $R$-module.

The form of every element in $V_{T}$ is illustrated in the following proposition.

\section{Proposition (2.2) [1]:}

If $S=\left\{V_{j}: j \in \Lambda\right\}$ is a basis for $\mathrm{V}$, then each element of $V_{T}$
Can be written in the form $\sum_{i=0}^{n} \quad \sum_{j \in \Lambda} c_{i j} T^{i} v_{j}$, where $c_{i j} \in F$

The symbol $\sum_{j \in \Lambda}$ means that the sum is taken over a finite subset of $\Lambda$.

\section{Remark (2.3) [1]:}

$\mathrm{V}_{\mathrm{I}}$ is a finitely generated R-module if and only if $\mathrm{V}$ is a finite dimensional vector space.

In this remark there is a relation between a finite dimensional vector space $\mathrm{V}$ and $V_{T}$

\section{Remark (2.4) [1]:}

Let $V$ be a finite dimensional vector space. Let $T$ be an operator on $V$, then $V_{T}$ is a finitely generated $R$ - module.

Recall that if $T$ and $S$ two operators on V.S is similar to $T$ if there exists an invertible operator $h$ on $V$ such that $h S h^{-1}=T$ [2].

\section{Proposition (2.5) [1]:}

Let $T$ and $S$ be two operators on $V$.Then $V_{S}$ is isomorphic to $V_{T}$ if and only if $S$ is similar to $T$.

\section{Definition (2.6) [2]:}

Let $T$ be an operator on a vector space. $T$ is said to be of finite rank if the image of $T$ is finite dimensional.

It is shown in (2.4) that if $V$ is a finite dimensional vector space, then $V_{T}$ is a finitely generated $R$ - module. Also if $V$ is finite dimensional vector space, and $T$ is any operator on $V$, then $T V$ is finite dimensional. Hence $T$ is of finite rank. Following proposition give the converse.

\section{Proposition (2.7) [1]:}

If $T$ is of finite rank, and $V_{T}$ is finitely generated, then $V$ is finite dimensional. 


\section{Definition (2.8) [3]:}

Let $T: V \rightarrow V$ be an operator. $v \in V$ is said to be an algebraic element (or $T$-algebraic) if there exists a non zero polynomial $P \in R$ such that $P(T) v=0$.

$T$ is said to be algebraic if there exists $P \neq$ 0 in $R$ such that $P(T) v=0, \forall v \in V$

\section{Proposition (2.9)[1]:}

Let $T: V \rightarrow V$ be an operator. Let $A=A(T)$ be the set of all $T$-algebraic elements. Then $A$ is a subspace of $V$.

There is a relation between the $T$-algebraic elements and the torsion elements of $V_{T}$ this relation is studied in the next proposition.

Recall that an element $\mathrm{m}$ of S-module where $S$ is a ring is torsion element if there exists $0 \neq t \in S$ such that $t m=0, M$ is torsion $S$-module if $\tau(M)=M$

where $\tau(M)$ the set of all torsion elements .[3]

\section{Proposition (2.10)[1]:}

Let $T$ be an operator on $V$, then $A_{T}=\tau\left(V_{T}\right)$

Recall that for any ring $S$ and any $S$-module $\quad M$, ann $(M)=\{t \in S: t m=$ $0, \forall m \in M\}$, and $\operatorname{ann}(M)=0$ then $M$ is a faithful $S$-module.[4].

\section{Proposition (2.11)[1]:}

$V_{T}$ is faithful $R-$ module if and only if $T$ is not an algebraic operator.

The module of the Unilateral shift operator is given finally.

Let $U: l_{2}(R) \rightarrow l_{2}(R)$ be the operator defined by $U\left(x_{1}, x_{2}, \ldots\right)=U\left(0, x_{1}, x_{2}, \ldots\right)$

This operator called the Unilateral shift operator.[5]

\section{$\frac{\text { Remark (2.12)[1]: }}{\forall i, K \in N,}$}

$\forall i, K \in N$, one can easily see that:

1. $\mathrm{U} \mathrm{e}_{\mathrm{k}}=\mathrm{e}_{\mathrm{k}+1} 2 \cdot \mathrm{U}^{\mathrm{i}} \mathrm{e}_{\mathrm{k}}=\mathrm{e}_{\mathrm{i}+\mathrm{k}} 3 \cdot \mathrm{U}^{\mathrm{i}} \mathrm{e}_{\mathrm{k}}=$ $\mathrm{U}^{\mathrm{i}+\mathrm{k}-1} \mathrm{e}_{1}$.

Recall that a left $\mathrm{R}$-module $\mathrm{M}$ is called acyclic if $\mathrm{M}$ can be generated by a single element. $M(x)=R x=\{r x / r \in R\}$ for some $x$ in $M$.

\section{Theorem (2.13)[1]:}

Let $U$ be the Unilateral shift operator on $H$. Then $H_{U}$ is a cyclic faithful $R$-module. Hence a free $R$-module.

\section{Main Results \\ Definition (3.1):}

Let $\mathrm{R}=\mathrm{F}[\mathrm{x}, \mathrm{y}]$ be the ring of polynomials in $\mathrm{X}, \mathrm{y}$ with coefficients in $\mathrm{F}$. Let $V$ be an inner product space over afield $F$ and $T$ be a bounded linear operator acting on the elements of $V$ on the left .We will define a left R-module on $\mathrm{V}$ as follows: $\Psi: R \times V \rightarrow V$

by $\Psi(P, v)=P\left(T, T^{*}\right) v \quad$ i.e $\quad \mathrm{P}(\mathrm{x}, \mathrm{y})=$ $\sum_{\mathrm{i}=0}^{\mathrm{m}} \quad \sum_{\mathrm{j}=0}^{\mathrm{n}} \quad \mathrm{a}_{\mathrm{ij}} \mathrm{x}^{\mathrm{i}} \mathrm{y}^{\mathrm{j}}, \mathrm{a}_{\mathrm{ij}} \in \mathrm{F}$. [6] It is clear that $\Psi$ makes $\mathrm{V}$ a unitary left $\mathrm{R}$-module. We shall denote this module by $\mathrm{V}_{\mathrm{T}, \mathrm{T}^{*}}$.

In the following proposition we introduce the form of each element of $\mathrm{V}_{\mathrm{T}, \mathrm{T}^{*}}$.

\section{Proposition (3.2):}

If $S=\left\{v_{l}: l \in \Lambda\right\}$ is a basis for $V$. then each element of $\mathrm{V}_{\mathrm{T}, \mathrm{T}^{*}}$ can be Written in the form $\sum_{j=0}^{m} \sum_{i=0}^{n} \sum_{l \in \Lambda} c_{i l} T^{i} T^{* j} v_{l}, c_{i l} \in F$

The symbol $\sum_{\mathrm{l} \in \Lambda}$ means that the sum is taken over a finite subset of $\Lambda$

Proof:- let $\mathrm{w} \in \mathrm{V}_{\mathrm{T}, \mathrm{T}^{*}}$, then

$\mathrm{w}=\sum_{\mathrm{k}=1}^{\mathrm{m}^{\prime}} \mathrm{P}_{\mathrm{k}} \cdot \mathrm{w}_{\mathrm{k}}$, where

$$
\mathrm{P}_{\mathrm{k}}(\mathrm{x}, \mathrm{y})=\sum_{\mathrm{j}=0}^{\mathrm{m}}\left(\mathrm{P}_{\mathrm{k}}(\mathrm{x})\right) \mathrm{y}^{\mathrm{j}} \text {, }
$$

$\mathrm{P}_{\mathrm{k}}(\mathrm{x})=\sum_{\mathrm{i}=0}^{\mathrm{n}_{\mathrm{k}}} \mathrm{a}_{\mathrm{ik}} \mathrm{x}^{\mathrm{i}}$

$\mathrm{P}_{\mathrm{k}}(\mathrm{x}, \mathrm{y})=\sum_{\mathrm{j}=0}^{\mathrm{m}}\left(\sum_{\mathrm{i}=0}^{\mathrm{n}_{\mathrm{k}}} \mathrm{a}_{\mathrm{ik}} \mathrm{x}^{\mathrm{i}}\right) \mathrm{y}^{\mathrm{j}} \in \mathrm{R}$

$w_{K}=\sum_{l \in \Lambda} b_{K l} v_{l} \in V$,then

$w=\sum_{k=1}^{m^{\prime}} \sum_{j=0}^{m}\left(\sum_{i=0}^{n_{k}} a_{i k} T^{i} T^{* j}\right)\left(\sum_{l \in \Lambda} b_{k l} v_{l}\right)$

Let $\mathrm{n}=\max \left\{\mathrm{n}_{1}, \mathrm{n}_{2}, \cdots, \mathrm{n}_{\mathrm{m}}\right\}, \mathrm{a}_{\mathrm{ik}}=0, \forall \mathrm{i}>$ $\mathrm{n}_{\mathrm{k}}, \mathrm{k}=1,2, \cdots, \mathrm{m}^{\prime}$

Then

$\mathrm{w}=$

$\sum_{\mathrm{j}=0}^{\mathrm{m}} \quad \sum_{\mathrm{i}=0}^{\mathrm{n}} \mathrm{a}_{\mathrm{ik}} \mathrm{T}^{\mathrm{i}} \mathrm{T}^{* \mathrm{j}}\left(\sum_{k=1}^{m^{\prime}} \quad \sum_{l \in \Lambda} b_{k l} v_{l}\right)$

$=\sum_{\mathrm{j}=0}^{\mathrm{m}} \quad \sum_{\mathrm{i}=0}^{\mathrm{n}} \mathrm{T}^{\mathrm{i}} \mathrm{T}^{* \mathrm{j}}\left(\sum_{l \in \Lambda} \sum_{k=1}^{m^{\prime}} a_{i k} b_{k l} v_{l}\right)$

$=\sum_{\mathrm{j}=0}^{\mathrm{m}} \quad \sum_{\mathrm{i}=0}^{\mathrm{n}} \mathrm{T}^{\mathrm{i}} \mathrm{T}^{* \mathrm{j}}\left(\sum_{l \in \Lambda} c_{i l} v_{l}\right)$

Where $c_{i l}=\sum_{k=1}^{m^{\prime}} a_{i k} b_{k l}$

Thus w $=\sum_{\mathrm{j}=0}^{\mathrm{m}} \quad \sum_{\mathrm{i}=0}^{\mathrm{n}} \quad \sum_{l \in \Lambda} c_{i l} T^{i} T^{*^{j}} v_{l}$

\section{Examples (3.3):}

1. Let $\left\{v_{l}: l \in \Lambda\right\}$ be abasis for an inner product space $\mathrm{V}$.

(a) Let 0 be the zero operator on $V$.If $w \in$ $\mathrm{V}_{0,0^{*}}$ then by proposition (3.2) 
$w=\sum_{j=0}^{m} \sum_{i=0}^{n} \sum_{l \in \Lambda} c_{i l} 0^{i} 0^{* j}, c_{i l} \in F$. Recall that $0^{0}=\mathrm{I}$, then $w=\sum_{l \in \Lambda} c_{0 l} v_{l}$

(b) Let I be the Identity operator on V.If $\mathrm{W} \in \mathrm{V}_{\mathrm{I}, \mathrm{I}^{*}}$ then by proposition (3.2)

,$w=\sum_{j=0}^{m} \sum_{i=0}^{n} \sum_{l \in \Lambda} c_{i l} I^{i} I^{j} v_{l}$

put $c_{l}=\sum_{i+j=0}^{n+m} c_{i l}$

Then $w=\sum_{l \in \Lambda} c_{l} v_{l}$

2. Let $\mathrm{T}$ be a bounded linear operator on a Hilbert space.

(a) $\mathrm{T}$ is a Self-adjoint operator,

if $\mathrm{T}=\mathrm{T}^{*}$.[2]

Then by proposition (3.2)

$$
w=\sum_{i+j=0}^{n+m} \sum_{l \in \Lambda} c_{i l} T^{i+j} v_{l}
$$

(b) $T$ is Normal operator , if $\mathrm{TT}^{*}=\mathrm{T}^{*} \mathrm{~T}$.[2]

Then by proposition (3.2),

$\mathrm{w}=\sum_{\mathrm{j}=0}^{\mathrm{m}} \quad \sum_{i=0}^{n} \quad \sum_{\boldsymbol{l} \in \Lambda} c_{i l} \mathrm{~T}^{*^{\mathrm{i}}} \mathrm{T}^{\mathrm{j}} v_{\boldsymbol{l}}$

\section{Remark (3.4):}

$\mathrm{V}_{\mathrm{I}, \mathrm{I}^{*}}$ is a finitely generated $R$-module if and only if $\mathrm{V}$ is a finite dimensional an inner product space.

\section{Proof:}

Let $\mathrm{V}_{\mathrm{I}, \mathrm{I}^{*}}$ is finitly generated $R$-module with generators $\left\{\mathrm{u}_{1}, \mathrm{u}_{2}, \ldots, \mathrm{u}_{\mathrm{m}}\right\}$ we prove by contradiction suppose that $\mathrm{V}$ is not finite

Dimensional. Let $\left\{e_{l}: l \in \Lambda\right\}$ be a basis for $\mathrm{V}$ by Ex :1.(b), $\mathrm{u}_{\mathrm{j}} \in \mathrm{V}$

$\mathrm{u}_{\mathrm{j}}=\sum_{\mathrm{k} \in \Lambda} \mathrm{c}_{\mathrm{k}} \mathrm{e}_{\mathrm{k}}, \mathrm{j}=1,2, \ldots, \mathrm{m}$. Thus $\mathrm{V}_{\mathrm{I}, \mathrm{I}^{*}}$ can be generated by a finite number of elements of the set $\left\{e_{l}: l \in \Lambda\right\}$, say, $\left\{\mathrm{e}_{1}, \mathrm{e}_{2}, \ldots, \mathrm{e}_{\mathrm{n}}\right\}$

Therefore if $K>n$ then $\mathrm{e}_{\mathrm{k}}=\sum_{\mathrm{t}=1}^{\mathrm{m}} \mathrm{P}_{\mathrm{t}} \cdot \mathrm{e}_{\mathrm{t}}$

Where $P_{t}(\mathrm{x}, \mathrm{y})=\sum_{i=0}^{n} \quad\left(\sum_{\mathrm{j}=0}^{\mathrm{k}_{\mathrm{t}}} \mathrm{a}_{\mathrm{tj}} \mathrm{x}^{\mathrm{j}}\right) \mathrm{y}^{\mathrm{i}}$

$\mathrm{e}_{\mathrm{k}}=\sum_{\mathrm{t}=1}^{\mathrm{m}} \sum_{\mathrm{i}=0}^{\mathrm{n}}\left(\sum_{\mathrm{j}=0}^{\mathrm{k}_{\mathrm{t}}} \mathrm{a}_{\mathrm{tj}} \mathrm{x}^{\mathrm{j}}\right) \mathrm{y}^{\mathrm{i}} \cdot \mathrm{e}_{\mathrm{t}}$

$$
=\sum_{j=0}^{k_{t}} a_{t j} e_{t}
$$

Put $\mathrm{a}_{\mathrm{t}}=\sum_{\mathrm{j}=0}^{\mathrm{k}_{\mathrm{t}}} \mathrm{a}_{\mathrm{tj}}$

then $p_{t}$. $e_{t}=a_{t} \cdot e_{t}, t=1,2, \ldots, m$

Therefore, $e_{k}=\sum_{t=1}^{n} a_{t} e_{t}$

Which is a contradiction, thus $\mathrm{V}$ is a finite dimensional an inner product space.

Assume $\mathrm{V}$ is an n-dimensional normed space with basis $\left\{\mathrm{v}_{1}, \mathrm{v}_{2}, \ldots, \mathrm{v}_{\mathrm{n}}\right\}$. Let $w \in V_{I, I^{*}}$ by Ex:1.(b) $\mathrm{w}=\sum_{l=1}^{n} c_{l} v_{l}$
This shows that $\mathrm{V}_{\mathrm{I}, \mathrm{I}^{*}}$ is a finitely generated $R$-module.

Compare the following with proposition (2-5)

\section{Proposition (3.5):}

Let $\mathrm{T}, \mathrm{S}$ be two bounded operators on V.then $\mathrm{V}_{\mathrm{S}, \mathrm{S}}$ and $\mathrm{V}_{\mathrm{T}, \mathrm{T}^{*}}$ are isomorphic $\mathrm{R}$-module iff $\mathrm{S}$ and $\mathrm{T}$ are similar.

\section{Proof:}

If $\mathrm{V}_{\mathrm{S}, \mathrm{S}^{*}}$ is isomorphic to $\mathrm{V}_{\mathrm{T}, \mathrm{T}^{*}}$

Let $h: V_{\mathrm{S}, \mathrm{S}^{*}} \rightarrow \mathrm{V}_{\mathrm{T}, \mathrm{T}^{*}}$ be an R-isomorphisim

Thus $h\left(w_{1}+w_{2}\right)=h\left(w_{1}\right)+$

$\mathrm{h}\left(\mathrm{w}_{2}\right), \forall \mathrm{w}_{1}, \mathrm{w}_{2} \in \mathrm{V}_{\mathrm{S}, \mathrm{S}^{*}}$

$\mathrm{h}(\mathrm{P}(\mathrm{x}, \mathrm{y}) \cdot \mathrm{w})=\mathrm{P}(\mathrm{x}, \mathrm{y}) \cdot \mathrm{h}(\mathrm{w}), \forall \mathrm{P} \in \mathrm{R}, \mathrm{w} \in$ $\mathrm{V}_{\mathrm{S}, \mathrm{S}^{*}}$

i.e $\mathrm{h}$ is homomorphisim then we can define $\mathrm{h}$ as:

$\mathrm{h}\left[\mathrm{P}\left(\mathrm{S}, \mathrm{S}^{*}\right) \mathrm{w}\right]=\mathrm{P}\left(\mathrm{T}, \mathrm{T}^{*}\right) \mathrm{h}(\mathrm{w})$

If $P$ is a constant polynomial $\mathrm{a}, \mathrm{a} \in \mathrm{F}$, then $\mathrm{h}(\mathrm{av})=\mathrm{ah}(\mathrm{v})$

Thus $h$ is a linear operator call it again $h$, if $P(x, y)=x+y$

Then $h(P(x, y) w)=P(x, y) h(w)$

$h((x+y) w)=(x+y) h(w)$

$\mathrm{h}\left(\mathrm{S}+\mathrm{S}^{*}\right)=\left(\mathrm{T}+\mathrm{T}^{*}\right) \mathrm{h}$

$\mathrm{hSh}^{-1}+\mathrm{hS}^{*} \mathrm{~h}^{-1}=\mathrm{h}^{-1} \mathrm{Th}+\mathrm{h}^{-1} \mathrm{~T}^{*} \mathrm{~h}$

Then $\mathrm{hSh}^{-1}=\mathrm{T}, \mathrm{hS}^{*} \mathrm{~h}^{-1}=\mathrm{T}^{*}$

Then $\mathrm{S}$ is similar to $\mathrm{T}$

If $\mathrm{S}$ and $\mathrm{T}$ are similar then there exists an operator h on V s.t

$\mathrm{h}\left(\mathrm{S}+\mathrm{S}^{*}\right) \mathrm{h}^{-1}=\mathrm{T}+\mathrm{T}^{*}$ it is easy to cheack that

$\mathrm{hP}\left(\mathrm{S}, \mathrm{S}^{*}\right)=\mathrm{P}\left(\mathrm{T}, \mathrm{T}^{*}\right) \mathrm{h} \forall \mathrm{P} \in \mathrm{R}$

Define $h^{\prime}: V_{S, S^{*}} \rightarrow V_{T, T^{*}}$

By $h^{\prime}\left[P\left(S, S^{*}\right) v\right]=P\left(T, T^{*}\right) h(v)$

If $\mathrm{P}_{1}\left(\mathrm{~S}, \mathrm{~S}^{*}\right) \mathrm{v}_{1}=\mathrm{P}_{2}\left(\mathrm{~S}, \mathrm{~S}^{*}\right) \mathrm{v}_{2}$

Then $h\left[\mathrm{P}_{1}\left(\mathrm{~S}, \mathrm{~S}^{*}\right) \mathrm{v}_{1}\right]=\mathrm{h}\left[\mathrm{P}_{2}\left(\mathrm{~S}, \mathrm{~S}^{*}\right) \mathrm{v}_{2}\right]$

(since $\mathrm{h}$ operator)

Then by

(1) $\mathrm{P}_{1}\left(\mathrm{~T}, \mathrm{~T}^{*}\right) \mathrm{h}\left(\mathrm{v}_{1}\right)=\mathrm{P}_{2}\left(\mathrm{~T}, \mathrm{~T}^{*}\right) \mathrm{h}\left(\mathrm{v}_{2}\right)$

By

(2) $\mathrm{h}^{\prime}\left[\mathrm{P}_{1}\left(\mathrm{~S}, \mathrm{~S}^{*}\right) \mathrm{v}_{1}\right]=\mathrm{h}^{\prime}\left[\mathrm{P}_{2}\left(\mathrm{~S}, \mathrm{~S}^{*}\right) \mathrm{v}_{2}\right]$.thus $\mathrm{h}^{\prime}$ is well define.

If $\mathrm{h}^{\prime}\left[\mathrm{P}\left(\mathrm{S}, \mathrm{S}^{*}\right) \mathrm{v}\right]=0$,

then $\mathrm{P}\left(\mathrm{T}, \mathrm{T}^{*}\right) \mathrm{h}(\mathrm{v})=0$

By (1) $h p\left(S, S^{*}\right) v=0$ but $h$ is invertible then $\mathrm{p}\left(\mathrm{S}, \mathrm{S}^{*}\right) \mathrm{v}=0$ 
Therefore $\mathrm{h}^{\prime}$ is $1-1$

Let $\mathrm{P}\left(\mathrm{T}, \mathrm{T}^{*}\right) \mathrm{v} \in \mathrm{V}_{\mathrm{T}, \mathrm{T}^{*}}$ since $\mathrm{v} \in \mathrm{V}$

Then $\mathrm{h}^{-1}(\mathrm{v}) \in \mathrm{V}$ and $\mathrm{P}\left(\mathrm{S}, \mathrm{S}^{*}\right) \mathrm{h}^{-1}(\mathrm{v}) \in \mathrm{V}_{\mathrm{S}, \mathrm{S}^{*}}$

$\mathrm{h}^{\prime}\left[\mathrm{P}\left(\mathrm{S}, \mathrm{S}^{*}\right) \mathrm{h}^{-1}(\mathrm{v})\right]=\mathrm{P}\left(\mathrm{T}, \mathrm{T}^{*}\right) \mathrm{hh}^{-1}(\mathrm{v})=$ $\mathrm{P}\left(\mathrm{T}, \mathrm{T}^{*}\right) \mathrm{v}$

Thus h' is on to

Note $h^{\prime}\left[P\left(S, S^{*}\right) v\right]=h\left[P\left(S, S^{*}\right) v\right]$, but $h$ is an operator, hence

$\mathrm{h}^{\prime}$ is an R-homomorphism, therefore $\mathrm{h}^{\prime}$ is an R-isomorphism.

\section{Remark (3.6):}

If $V$ is a finite dimensional an inner product space, then $V_{T, T^{*}}$ is finitely generated R-module.

We show in (3.6) that if $\mathrm{V}$ is a finite dimensional an inner product space, then $V_{T}$ is finitely generated R-module, also if $V$ is finite dimensional and $T$ is any operator on $V$, then $T V$ is finite dimensional, hence $T$ is of finite rank.

\section{Proposition (3.7):}

If $\mathrm{T}$ is of finite rank, and $\mathrm{V}_{\mathrm{T}, \mathrm{T}^{*}}$ is finitely generated, then $\mathrm{V}$ is finite dimensioal.

\section{Proof:}

Let $\mathrm{K}=\mathrm{K}\left(\mathrm{T} \mathrm{T}^{*}\right)=\left\{\mathrm{w} \in \mathrm{V}: \mathrm{TT}^{*} \mathrm{w}=0\right\}$ it is clear that $\mathrm{K}$ is an invariant subspaces of $\mathrm{V}$, and $\mathrm{TT}^{*} \mathrm{~V} \cong \frac{\mathrm{V}}{\mathrm{K}}$

We prove by contradiction way .Assume $\mathrm{V}$ is not finite dimensional. $\mathrm{TT}^{*} \mathrm{~V}$ is finite dimensional since $\mathrm{T}$ is finite rank,thus $\mathrm{K}$ must be infinite dimensional but $\mathrm{K}$ is an invariant subspace of $V$,then the submodule $K_{T, T^{*}}$ is generated by the set $\left\{\mathrm{T}^{\mathrm{i}} \mathrm{T}^{*} \mathrm{w}_{\mathrm{l}}: l \in \Lambda\right.$; $\mathrm{i}=0,1, \cdots ; \mathrm{j}=0,1, \cdots\}$ where $\left\{\mathrm{w}_{\mathrm{l}}: \mathrm{l} \in \Lambda\right\}$ is abasis for $K \cdot w_{l} \in \mathrm{k}$ means that $\mathrm{T} \mathrm{T}^{*} \mathrm{w}_{\mathrm{l}}=$ 0 .Hence the restriction of $\mathrm{T} \mathrm{T}^{*}$ on $\mathrm{K}$ is the zero operator,thus $\mathrm{K}_{\mathrm{T}, \mathrm{T}^{*}}=\mathrm{K}_{0,0^{*}}$ by (3.2) $\mathrm{K}_{\mathrm{T}, \mathrm{T}^{*}}$ cannot be finitely generated, and since $\mathrm{R}$ Noetherian [7], $\mathrm{V}_{\mathrm{T}, \mathrm{T}^{*}}$ is finitely generated then $\mathrm{K}_{\mathrm{T}, \mathrm{T}^{*}}$ is finitely generated this contradiction shows that $\mathrm{V}$ is finite dimensional.

\section{Definition (3.8) [8]:}

An operator $\mathrm{T} \in \mathrm{B}(\mathrm{H})$ is said to be *-algebraic operator if there exists non-zero polynomial of two variables $P$ such that
$\mathrm{P}\left(\mathrm{T}, \mathrm{T}^{*}\right) \mathrm{x}=0 \quad, \forall x \in H . \quad x \in H \quad$ is called $*$-algebraic element if there exists non zero polynomial of two variables $P$ such that $\mathrm{P}\left(\mathrm{T}, \mathrm{T}^{*}\right) \mathrm{x}=0$.

\section{Proposition (3.9):}

Let $\mathrm{T}: \mathrm{H} \rightarrow \mathrm{H}$ and $\mathrm{A}=\mathrm{A}\left(\mathrm{T}, \mathrm{T}^{*}\right)$ be the set of all *-algebraic elements then $\mathrm{A}$ is a subspace of $\mathrm{H}$.

\section{Proof:}

Let $u, v \in A$ then there exist non-zero polynomial $\mathrm{p}, \mathrm{q}$ inR such that

$\mathrm{P}\left(\mathrm{T}, \mathrm{T}^{*}\right) \mathrm{u}=0$ and $\mathrm{q}\left(\mathrm{T}, \mathrm{T}^{*}\right) \quad \mathrm{v}=0, \quad$ then $\mathrm{P}\left(\mathrm{T}, \mathrm{T}^{*}\right) \mathrm{q}\left(\mathrm{T}, \mathrm{T}^{*}\right)(\mathrm{u}+\mathrm{v})=0$

Since $\mathrm{R}=\mathrm{F}[\mathrm{x}, \mathrm{y}]$ is an integral domain [9], hence $\mathrm{Pq} \neq 0$, therefore

$\mathrm{u}+\mathrm{v} \in \mathrm{A}$ if $\mathrm{a} \in \mathrm{F}$ then $\mathrm{P}\left(\mathrm{T}, \mathrm{T}^{*}\right) \mathrm{au}=$ $\mathrm{aP}\left(\mathrm{T}, \mathrm{T}^{*}\right) \mathrm{u}=0$ thus $\mathrm{au} \in \mathrm{A}$ therefore $\mathrm{A}$ subspace of $\mathrm{H}$.

\section{Proposition (3.10):}

Let $\mathrm{T}$ be an operator on $\mathrm{H}$, then $\mathrm{A}_{\mathrm{T}, \mathrm{T}^{*}}=\tau\left(\mathrm{H}_{\mathrm{T}, \mathrm{T}^{*}}\right)$

\section{Proof:}

let $0 \neq w \in A_{T, T^{*}}$. then $w=\sum_{i=0}^{n} P_{i} \quad v_{i}$ for some $\mathrm{P}_{\mathrm{i}} \in \mathrm{R}, \mathrm{v}_{\mathrm{i}} \in A \forall \mathrm{i}$

There exists $q_{i} \neq 0$ in $R$ such that $\mathrm{q}_{\mathrm{i}}\left(\mathrm{T}, \mathrm{T}^{*}\right) \mathrm{v}_{\mathrm{i}}=0$

henceq $\left(\mathrm{T}, \mathrm{T}^{*}\right) \mathrm{w}=\mathrm{q} \cdot \mathrm{w}=0$ where $\mathrm{q}=$ $\mathrm{q}_{1} \mathrm{q}_{2} \cdots \mathrm{q}_{\mathrm{n}}$ Thus $\mathrm{w} \in \tau\left(\mathrm{H}_{\mathrm{T}, \mathrm{T}^{*}}\right)$

And let $u \in \tau(H)$, then there exists $P \neq 0$ in $R$ Such that P. $\mathrm{u}=0$ therefore $\mathrm{P}\left(\mathrm{T}, \mathrm{T}^{*}\right) \mathrm{u}=$ 0 , thus $\mathrm{u} \in \mathrm{A}_{\mathrm{T}, \mathrm{T}^{*}}$

Therefore $A_{T, T^{*}}=\tau\left(H_{T, T^{*}}\right)$

In the following proposition we give the relation between faithful R-module and *-algebraic operator.

\section{Proposition (3.11):}

$\mathrm{H}_{\mathrm{T}, \mathrm{T}^{*}}$ is a faithful $R$-module if and only if $T$ is not $*$-algebraic operator.

\section{Proof:}

Let $\mathrm{P} \in \mathrm{R}$ such that $\mathrm{P}\left(\mathrm{T}, \mathrm{T}^{*}\right) \mathrm{v}=0 \forall \mathrm{v} \in \mathrm{H}$

Then P. $v=0 \forall x \in H$. Thus P. $v=0 \forall \mathrm{v} \in$ $\mathrm{H}_{\mathrm{T}, \mathrm{T}^{*}}$ hence $\mathrm{P} \in \operatorname{ann}\left(\mathrm{H}_{\mathrm{T}, \mathrm{T}^{*}}\right)$

Therefor $P=0$ and $T$ is not $*_{\text {-algebraic }}$ operator.

Conversely, let $\mathrm{P} \in$ ann $\left(\mathrm{H}_{\mathrm{T}, \mathrm{T}^{*}}\right)$ 
Then $\mathrm{P} . \mathrm{v}=0 \forall \mathrm{v} \in \mathrm{H}_{\mathrm{T}, \mathrm{T}^{*}}$, thus $\mathrm{P}\left(\mathrm{T}, \mathrm{T}^{*}\right) \mathrm{v}=$ $0 \forall \mathrm{v} \in \mathrm{H}$

If $T$ is not $*$-algebraic operator, then $\quad \mathrm{P}=$ 0 . Therefor $\mathrm{H}_{\mathrm{T}, \mathrm{T}^{*}}$ is faithful.

Finally, we study the module of Unilateral shift operator in the following.

\section{Theorem (3.12) :}

Let $\mathrm{U}$ be the Unilateral shift operator on $\mathrm{H}$. then $\mathrm{H}_{\mathrm{U}, \mathrm{U}^{*}}$ is a cyclic $R$-module hence a free $R$-module.

\section{Proof:}

Let $\mathrm{w} \in \mathrm{H}_{\mathrm{U}, \mathrm{U}^{*}}$, then

$$
w=\sum_{l=1}^{m^{\prime}} \sum_{j=0}^{m} \sum_{i=0}^{n} a_{i l} U^{i} U^{* j} e_{l}
$$

SinceU $^{*}=\mathrm{B}, \mathrm{w}=$

$\sum_{l=1}^{m^{\prime}} \sum_{j=0}^{m} \sum_{i=0}^{n} a_{i l} U^{i} B^{j} e_{l} w=$

$\sum_{l=1}^{m^{\prime}} \sum_{j=0}^{m} \quad \sum_{i=0}^{n} a_{i l} U^{i} e_{l-j} \cdot[1]$

$\sum_{l=1}^{m^{\prime}} \sum_{j=0}^{m} \sum_{i=0}^{n} a_{i l} U^{i+l-1} U^{-j} e_{1}$. By(

remark 3 ,

Thus $\mathrm{w}=$ P. $\mathrm{e}_{1}$,

where

$\sum_{l=1}^{m^{\prime}} \sum_{j=0}^{m} \sum_{i=0}^{n} a_{i l} x^{i+l-1} y^{j}$

$$
P(x, y)=
$$

Therefore $\mathrm{H}_{\mathrm{U}, \mathrm{U}^{*}}$ is cyclic R-module generated by $\mathrm{e}_{1}$.thus $H_{U, U^{*}}$ is afree $R$-module. [10]

\section{Corollary (3.13):}

Let $\mathrm{U}$ be the unilateral shift operator on $\mathrm{H}$. then $H_{U, U^{*}}$ is a faithful $R$-module.

\section{Proof:}

Let

$\mathrm{P}(\mathrm{x}, \mathrm{y})=\sum_{\mathrm{i}=0}^{\mathrm{m}} \quad \sum_{\mathrm{j}=0}^{\mathrm{n}} \quad \mathrm{a}_{\mathrm{ij}} \mathrm{x}^{\mathrm{i}} \mathrm{y}^{\mathrm{j}} \in \operatorname{ann}\left(\mathrm{H}_{\mathrm{U}, \mathrm{U}^{*}}\right)$

then $P(x, y) \cdot e_{1}=0$

Hence

$\sum_{\mathrm{i}=0}^{\mathrm{m}} \sum_{\mathrm{j}=0}^{\mathrm{n}} \mathrm{a}_{\mathrm{ij}} \mathrm{U}^{\mathrm{i}} \mathrm{B}^{\mathrm{j}} \mathrm{e}_{1}=$

$0, \sum_{\mathrm{i}=0}^{\mathrm{m}} \quad \sum_{\mathrm{j}=0}^{\mathrm{n}} \mathrm{a}_{\mathrm{ij}} \mathrm{U}^{\mathrm{i}}\left(\mathrm{e}_{1-\mathrm{j}}\right)=0$.[1]

By (2.12) remark 2 we have $\sum_{\mathrm{i}=0}^{\mathrm{m}} \quad \sum_{\mathrm{j}=0}^{\mathrm{n}} \quad \mathrm{a}_{\mathrm{ij}} \mathrm{e}_{\mathrm{i}-\mathrm{j}+1}=0$.

But $e_{1}, e_{2}, \ldots, e_{m-n+1}$ are linearly Independent hence $\mathrm{a}_{\mathrm{ij}}=0$

$\forall \mathrm{i}=0,1, \ldots, \mathrm{m}, j=0,1, \ldots, \mathrm{n}$ thus $P=0$

Therefore $\mathrm{H}_{\mathrm{U}, \mathrm{U}^{*}}$ is a faithful R-module.

\section{References}

[1] Salma M Faris, Linear Operators and Modules, A master thesis, College of Science Baghdad, 1994
[2] Sterling K. Berberian, Introduction to Hilbert Space, Chelsa publishing company, New York N.Y, 1961.

[3] Kaplansky I, Infinite abelian groups, The University of Michigan press. Ann Arbor, 1962.

[4] Ahmad Yousefian Darani, Notes on Annihilator conditions in Modules over commutative Rings, An. St. Univ. Ovidius Constanta, 2010.

[5] Halmos P.R, A Hilbert space problem book, springer verlag, New York, Heudelberg Berlin, 1974.

[6] David M Burton, Abstract and linear Algebra, University of Hampshire, 283, 1972.

[7] Hilary Term, Integral Domains, Modules and Algebraic Integers D.R. Wilkins, 2012.

[8] Samira Naji Kadhim, Reflexive Operators on Hilbert Space, Adoctor thesis ,College of Science Baghdad, 2005.

[9] Hilary Term, Integral Domains, Modules and Algebraic Integers Section 2, D.R. Wilkins, 2014.

[10] Kasch F, Modules and rings, Academic Press. London, 1982.

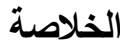

الموديول التابع للمؤثر T. الذي يرمز له بالرمز أعطي, عندما V فضاء الجداء الداخلي وT مؤثر خطي مقيد على V. سندرس في هذا البحث صفات للمؤثر T التي تؤثر على V V V العكس. 